\title{
Evaluation of Early Postoperative Intravenous Opioid Rescue as a Novel Quality Measure in Patients who Receive Thoracic Epidural Analgesia: A Retrospective Cohort Analysis and Prospective Performance Improvement Intervention
}

Nadav Levy ( $\square$ nlevy@bidmc.harvard.edu )

Beth Israel Deaconess Medical Center https://orcid.org/0000-0002-8236-4881

\section{Peter Santer}

Beth Israel Deaconess Medical Center

Liana Zucco

Beth Israel Deaconess Medical Center

\section{Sarah Nabel}

Beth Israel Deaconess Medical Center

Galina Korsunsky

Beth Israel Deaconess Medical Center

Satya Krishna Ramachandran

Beth Israel Deaconess Medical Center

\section{Research article}

Keywords: Thoracic epidural analgesia, Regional Anesthesia, Efficiency metrics, Quality improvement, Perioperative analgesia,

Posted Date: October 8th, 2020

DOl: https://doi.org/10.21203/rs.3.rs-86729/v1

License: (c) (1) This work is licensed under a Creative Commons Attribution 4.0 International License. Read Full License

Version of Record: A version of this preprint was published at BMC Anesthesiology on April 19th, 2021. See the published version at https://doi.org/10.1186/s12871-021-01332-7. 


\section{Abstract}

Background: In this study, we explored the prevalence of intravenous opioid rescue analgesia in the postanesthesia care unit (PACU-OpResc) as a single marker of thoracic epidural analgesia (TEA) failure and evaluated the resource implications and quality improvement applications of this measure.

Methods: We performed a retrospective analysis of all TEA placements during 2016-2018 at a single academic medical center in Boston, Massachusetts. The study exposure was the need for intravenous opioid rescue analgesia in the PACU (PACU-OpResc). Primary outcome was PACU length of stay (LOS). Secondary outcomes included delayed PACU discharge and intraoperative hypotension. The analyses were adjusted for confounding variables including patient comorbidities, surgical complexity, intraoperative intravenous opioids, chronic opioid use and local anesthetic bolus through TEA catheter. Post analysis chart review was conducted to determine the positive predictive value (PPV) of PACUOpResc for inadequate TEA. As a first Plan-Do-Study-Act cycle, we then introduced a checkbox for documentation of a sensory level check (SLC) after TEA placement. Post implementation data was collected for 7 months.

Results: PACU-OpResc was required by 211 (22.1\%) patients who received preoperative TEA, was associated with longer PACU LOS (incidence rate ratio 1.20, 95\% Cl:1.07-1.34, p=0.001) and delayed discharge due to inadequate pain control (odds ratio $5.15,95 \% \mathrm{Cl} 3.51-7.57, \mathrm{p}<0.001$ ). PACU-OpResc had a PPV of $76.3 \%$ and $60.4 \%$ for re-evaluation and manipulation of the TEA catheter in PACU, respectively. Following implementation of a checkbox, average monthly compliance with documented SLCs after TEA placement was noted to be $39.7 \%$. During this time, a reduction of $8.2 \%$ in the rate of PACU-OpResc was observed.

Conclusions: This study demonstrates that PACU-OpResc can be used as a quality assurance measure or surrogate for TEA efficiency, to track performance and monitor innovation efforts aimed at improving analgesia, such as our intervention to facilitate SLCs and reduced PACU-OpResc.

\section{Background}

Thoracic epidural analgesia (TEA) is a widely accepted and effective modality for postoperative pain management after thoracic and abdominal surgery. $(1,2)$ The specific benefits of TEA include better quality of pain control, decreased incidence of respiratory complications and reduced post-operative nausea and vomiting when compared to parenteral opioids. $(1,3-6)$ Thoracic epidural analgesia has a reported failure rate of up to $32 \%,(7)$ but the definition of 'failure' varies significantly in the literature. $(8,9)$ Several factors can be attributed to thoracic epidural failure, including technical (catheter placement, equipment), patient-related (difficult anatomy) or pharmacological (drugs and doses) causes. $(10,11)$ Furthermore, the placement and management of TEA is complex from a work-flow perspective and requires time, resources and expertise across several domains of care. The high risk of failure makes TEA a high-yield patient-centered care area for performance improvement. 
Traditionally, quality improvement efforts have focused on methods to streamline the TEA placement process, limit delays related to TEA placement on the overall operating room workflow and improve compliance with intraoperative epidural infusion.(10-14) While most of these improvement measures address local departmental issues, there are limited data on TEA surveillance at a system-level. We therefore conducted a retrospective observational study to explore the prevalence of intravenous opioid rescue analgesia in the post-anesthesia care unit (PACU-OpResc) in patients with TEA and evaluated the feasibility of this measure as a marker of TEA failure.

\section{Methods}

\section{Aim:}

We hypothesized that patients with TEA requiring postoperative rescue opioids in the PACU experienced prolonged lengths of stay in the PACU due to inadequate pain control. Our aim therefore was to evaluate the use of PACU-OpResc, as a patient centered metric for surveillance of TEA effectiveness, to pilot an intervention to target improvement in this patient population.

Furthermore, demonstrating a sensory block after a local anesthetic bolus is a common and recommended method to verify correct placement of TEA catheter $(9,11,13)$ that may reduce or aid in resolving cases of improper TEA placements. However, due to time constrains, this is often omitted preoperatively or performed postoperatively. $(13,15)$ Therefore in the second phase of the study, we hypothesized that an intervention to encourage the performance and recording of a sensory level check after TEA placements may reduce PACU-OpResc rates.

\section{Setting:}

Eligible patients undergoing thoracic or major abdominal surgery at Beth Israel Deaconess Medical Center (BIDMC), Boston, Massachusetts are routinely offered TEA for intra/postoperative pain management. Approximately 400 TEAs are placed pre-operatively at BIDMC yearly. Thoracic epidurals are routinely sited either by the anesthesiologist assigned to the case or by a member of the Acute Pain Service (APS), pending availability. The APS is a designated team of anesthesiologists and rotating residents who are also responsible for the post-operative management of all TEAs. A detailed description of the TEA placement process is included in appendix 1.

We performed a retrospective review of all surgical cases performed at BIDMC during the years 20162018 that were documented in the electronic health record (Phase 1) and a performance improvement intervention (Phase 2).

\section{Phase 1: Retrospective Cohort Analysis}

\section{Study Design}


Data collected for each patient included age, body mass index (BMI), the duration and type of surgical procedure and study specific intra and post-operative variables described below. Surgical complexity was defined by the work relative value units (work-RVUs) for each case. Intraoperative and postoperative data included the administration of IV opioids (morphine equivalents), adjunct analgesic medications, local anesthetic boluses and infusions via the TEA. Opioid prescriptions during the 90 days prior to surgery were reviewed as way to identify patients at higher risk of PACU delays due to pain.

\section{Study population}

The study population included all surgical patients at BIDMC between January 2016 and December 2018 for whom a TEA catheter was placed prior to a surgical procedure. Patients directly admitted to an intensive care unit from the operating room without intermediate stay in the PACU were excluded. Patients with missing data for confounder variables were excluded for complete-case analysis.

\section{Exposure}

The exposure was defined as a binary variable indicating any requirement for IV opioid administration during the PACU stay.

\section{Outcomes}

The primary outcome was defined as PACU length of stay (LOS) in minutes. Secondary outcomes included reasons for PACU delay as recorded by the PACU nurses upon discharge and measures of intraoperative hemodynamic stability as reflected by low blood pressure values. The latter was defined by frequency and duration of mean blood pressure below $65 \mathrm{mmHg}$ and $55 \mathrm{mmHg}$.

\section{Covariate model}

Based on clinical plausibility and available literature, analyses were adjusted for the following patientspecific covariates: age, BMI, 90-day pre-operative opioid prescription, and history of drug abuse. Case specific confounders included the duration of surgery, work relative value units (RVU), total dose of intraoperatively administered opioids (morphine equivalents), local anesthetic TEA boluses, TEA infusion duration and a high risk of postoperative pulmonary complications (i.e. Score for Prediction of Postoperative Respiratory Complications $[S P O R C] \geq 7)$.(16)

\section{Statistical analyses}

Negative binomial regression was used to assess the association between the dichotomized exposure and PACU length of stay as well as duration of hypotension. For binary endpoints, we employed multivariable logistic regression. Statistical significance was assumed at a $P$ value $<0.05$ for the primary analysis. Statistical analyses were performed using Stata (version 15; StataCorp LLC, College Station, $\mathrm{TX})$.

We conducted a post analysis chart review of all APS notes for the exposure group to estimate the sensitivity of our measure. We calculated the positive predictive value of PACU-OpResc for documented APS consult during PACU stay and for any documented intervention to improve analgesia (e.g. epidural catheter manipulation, replacement, bolus, additional IV medication or opioid infusion). 


\section{Phase 2: Performance Improvement Intervention}

\section{Intervention implementation}

We implemented an intervention using the Model for Improvement(17) and through a structured, first Plan-Do-Study-Act (PDSA) cycle, we introduced a sensory level check (SLC) discrete binary documentation element in the electronic procedure note (Compurecord, Phillips Healthcare, Andover, MA, USA). The checkbox provided an area in which to document whether an adequate, bilateral sensory band was achieved after TEA placement. The SLC checkbox was added to the digital procedure chart in May 2019, and its implementation was communicated to the APS team in a formal presentation, introducing the documentation change. Our intervention study measures were defined as SLC documentation (process measure) and PACU-OpResc (outcome measure). Following implementation, we collected data for the months of May to November 2019.

\section{Statistical analysis}

A 3-sigma statistical process control " $\mathrm{P}$ " chart was used to monitor and analyze the monthly average PACU-OpResc exposure. Rates of PACU-OpResc exposure were compared using a Likelihood ratio ChiSquare test. Data for the performance improvement study data were analyzed using JMP $B$ Pro (Version 14.0.0. SAS Institute Inc., Cary, NC,1989-2019). This manuscript adheres to the applicable STROBE(18) and SQUIRE(19) checklists (Phase 1 and Phase 2, respectively).

\section{Results}

\section{Phase 1: Retrospective Cohort Analysis}

\section{Study Cohort Characteristics}

A total of 1064 TEA catheters were placed for surgical patients at BIDMC between January 2016 to December 2018. After excluding 105 patients who were admitted directly to intensive care units and 5 patients for whom data was missing for confounder variables, our final study cohort included 954 TEA placements (Fig. 1). Among patients with pre-operative TEA, 211 (22.1\%) were exposed to PACU-OpResc. The study population characteristics and procedural data (TEA placement) are presented in Table 1. 
Table 1

Baseline characteristics of study population.

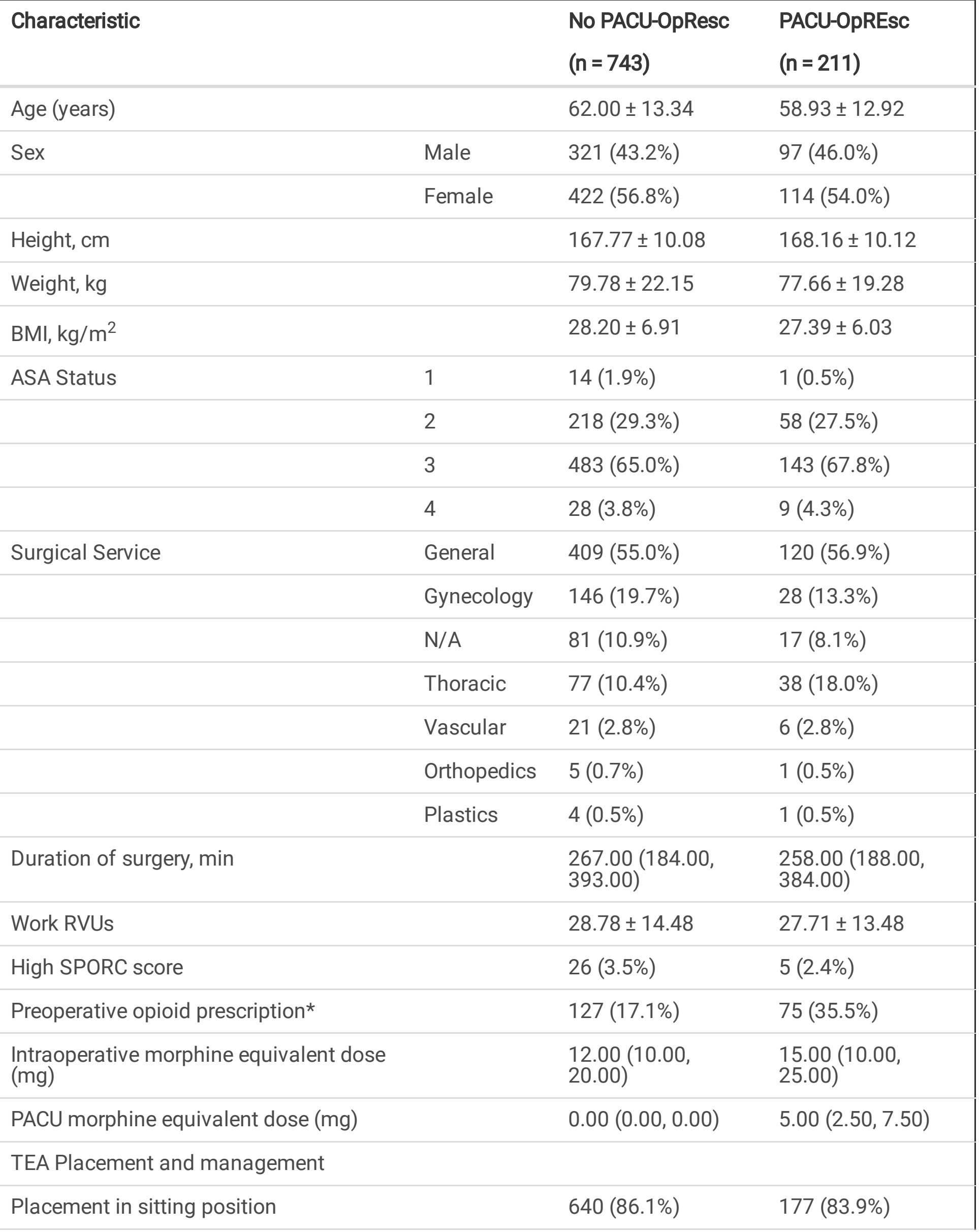




\begin{tabular}{|c|c|c|c|}
\hline Characteristic & & $\begin{array}{l}\text { No PACU-OpResc } \\
(\mathrm{n}=743)\end{array}$ & $\begin{array}{l}\text { PACU-OpREsc } \\
(\mathrm{n}=\mathbf{2 1 1})\end{array}$ \\
\hline \multicolumn{2}{|l|}{ Midline Approach } & $322(43.3 \%)$ & $87(41.2 \%)$ \\
\hline \multicolumn{2}{|l|}{ Local anesthetic bolus $y / n$} & $182(24 \%)$ & $66(31 \%)$ \\
\hline \multirow[t]{3}{*}{ After hours } & 0 & $636(85.6 \%)$ & $174(82.5 \%)$ \\
\hline & 1 & $16(2.2 \%)$ & $12(5.7 \%)$ \\
\hline & N/A & $91(12.2 \%)$ & $25(11.8 \%)$ \\
\hline \multicolumn{2}{|l|}{ First Case } & $361(48.6 \%)$ & $100(47.4 \%)$ \\
\hline \multirow[t]{4}{*}{ Placement Attempts } & 1 & $402(54.1 \%)$ & $103(48.8 \%)$ \\
\hline & 2 & $163(21.9 \%)$ & $45(21.3 \%)$ \\
\hline & $\geq 3$ & $88(11.8 \%)$ & $38(17.9 \%)$ \\
\hline & N/A & $90(12.1 \%)$ & $25(11.8 \%)$ \\
\hline \multicolumn{2}{|l|}{ Negative Test Dose } & $651(87.6 \%)$ & $184(87.2 \%)$ \\
\hline \multicolumn{2}{|l|}{ Placement duration, min } & $13.00 \pm 8.30$ & $13.72 \pm 9.45$ \\
\hline \multicolumn{2}{|l|}{$\begin{array}{l}\text { Incision to epidural infusion start time } \\
\text { (minutes) }\end{array}$} & $27.60 \pm 71.74$ & $31.83 \pm 74.59$ \\
\hline \multicolumn{2}{|l|}{$\begin{array}{l}\text { Epidural infusion during }>50 \% \text { of case } \\
\text { time }\end{array}$} & $603(81.2 \%)$ & $146(69.2 \%)$ \\
\hline \multicolumn{4}{|c|}{$\begin{array}{l}\text { Data are expressed as mean } \pm \text { standard deviation, frequency (prevalence in \%) or median (interquartile } \\
\text { range ( } 25 \text { th- } 75 \text { th percentile), values separated by comma). }{ }^{\star} \text { Opioid prescription within } 90 \text { days prior to } \\
\text { surgery. }\end{array}$} \\
\hline \multicolumn{4}{|c|}{$\begin{array}{l}\text { ASA: American Society of Anesthesiologist; BMI: Body Mass Index; PACU: Post Anesthesia Care Unit; } \\
\text { PACU-OpResC: intravenous opioid rescue analgesia in the post-anesthesia care unit; RVUs: Relative } \\
\text { Value Units; SPORC: Score of Prediction of postOperative Respiratory complications; TEA: Thoracic } \\
\text { Epidural Analgesia; }\end{array}$} \\
\hline
\end{tabular}

\section{Primary Outcome}

In patients exposed to PACU-OpResc, the median (IQR) PACU LOS was $286(221,427)$ min compared to $269(194,381) \mathrm{min}$ in patients who did not require PACU-OpResc. After adjusting for confounders, PACUOpResc was significantly associated with prolonged PACU length of stay (adjusted incidence rate ratio (alRR) $1.20,95 \% \mathrm{Cl} 1.07-1.34, \mathrm{p}=0.001$ ).

\section{Secondary Outcomes}


A total of 128 (60.66\%) patients exposed to PACU-OpResc experienced PACU delays for any reason compared to $283(38.09 \%)$ delays in patients not requiring rescue opioids. Exposure to PACU-OpResc was significantly associated with overall delay of discharge from the PACU (adjusted odds ratio (aOR) 2.57, $95 \% \mathrm{Cl} 1.83-3.60, \mathrm{p}<0.001)$. PACU discharge delays due to pain occurred significantly more often in patients exposed to PACU-OpResc: $41.23 \%$ of patients in the PACU-OpResc group were delayed due to pain compared to only $12.11 \%$ in the no PACU-OpResc group. The need for rescue opioids was significantly associated with pain-related PACU discharge delays (aOR 5.15, 95\% $\mathrm{Cl} 3.51-7.57, \mathrm{p}<0.001$ ). Exposure to PACU-OpResc was not associated with hemodynamic compromise as expressed by the duration of intraoperative hypotension, defined as a mean arterial pressure below 65 or $55 \mathrm{mmHg}$. Outcome data are presented in Table 2. 
Table 2

Association of PACU-OpResc with primary and secondary endpoints.

\begin{tabular}{|c|c|c|c|c|c|c|}
\hline \multirow[t]{2}{*}{ Outcomes } & \multirow{2}{*}{$\begin{array}{l}\text { No PACU- } \\
\text { OpResc } \\
(n=743)\end{array}$} & \multirow{2}{*}{$\begin{array}{l}\text { PACU- } \\
\text { OpResc } \\
(n=211)\end{array}$} & \multicolumn{2}{|c|}{ Unadjusted analysis } & \multicolumn{2}{|c|}{ Adjusted analysis } \\
\hline & & & $\begin{array}{l}\text { OR/IRR } \\
(95 \% \mathrm{Cl})\end{array}$ & $\begin{array}{l}\mathrm{p}- \\
\text { value }\end{array}$ & $\begin{array}{l}\text { OR/IRR } \\
\text { (95\% Cl) }\end{array}$ & $\begin{array}{l}\mathrm{p}- \\
\text { value }\end{array}$ \\
\hline \multicolumn{7}{|l|}{ Primary Outcome } \\
\hline $\begin{array}{l}\text { PACU length of stay, } \\
\text { min }\end{array}$ & $\begin{array}{l}269(194 \\
381)\end{array}$ & $\begin{array}{l}286(221 \\
427)\end{array}$ & $\begin{array}{l}1.21(1.08, \\
1.35)\end{array}$ & 0.001 & $\begin{array}{l}1.20(1.07 \\
1.34)\end{array}$ & 0.001 \\
\hline \multicolumn{7}{|l|}{ Secondary Outcomes } \\
\hline \multicolumn{7}{|l|}{ PACU discharge delay } \\
\hline Any reason, n (\%) & $\begin{array}{l}283 \\
(38.09 \%)\end{array}$ & $\begin{array}{l}128 \\
(60.66 \%)\end{array}$ & $\begin{array}{l}2.51(1.83 \\
3.43)\end{array}$ & $<.001$ & $\begin{array}{l}2.57(1.83 \\
3.60)\end{array}$ & $\begin{array}{l}<.001 \\
0.00\end{array}$ \\
\hline Pain, n (\%) & $90(12.11 \%)$ & $\begin{array}{l}87 \\
(41.23 \%)\end{array}$ & $\begin{array}{l}5.09(3.58 \\
7.24)\end{array}$ & $<.001$ & $\begin{array}{l}5.15(3.51, \\
7.57)\end{array}$ & <. 001 \\
\hline Cardiovascular, n (\%) & $\begin{array}{l}125 \\
(16.82 \%)\end{array}$ & $\begin{array}{l}42 \\
(19.91 \%)\end{array}$ & $\begin{array}{l}1.23(0.83, \\
1.81)\end{array}$ & 0.299 & $\begin{array}{l}1.34(0.88, \\
2.04)\end{array}$ & 0.175 \\
\hline PONV, n (\%) & $5(0.67 \%)$ & $3(1.42 \%)$ & $\begin{array}{l}2.13(0.50, \\
8.98)\end{array}$ & 0.304 & $\begin{array}{l}1.76(0.34, \\
8.96)\end{array}$ & 0.498 \\
\hline $\begin{array}{l}\text { Sedation/Respiratory, } \\
\text { n (\%) }\end{array}$ & 65 (8.75\%) & $\begin{array}{l}22 \\
(10.43 \%)\end{array}$ & $\begin{array}{l}1.21(0.73, \\
2.02)\end{array}$ & 0.455 & $\begin{array}{l}1.01(0.57 \\
1.80)\end{array}$ & 0.978 \\
\hline Voiding, n (\%) & $51(6.86 \%)$ & $\begin{array}{l}23 \\
(10.90 \%)\end{array}$ & $\begin{array}{l}1.66(0.99, \\
2.79)\end{array}$ & 0.055 & $\begin{array}{l}1.63(0.93 \\
2.85)\end{array}$ & 0.085 \\
\hline \multicolumn{7}{|l|}{$\begin{array}{l}\text { Hypotension MAP }< \\
65 \mathrm{mmHg}\end{array}$} \\
\hline Frequency, n (\%) & $\begin{array}{l}710 \\
(95.56 \%)\end{array}$ & $\begin{array}{l}201 \\
(95.26 \%)\end{array}$ & $\begin{array}{l}0.93(0.45, \\
1.93)\end{array}$ & 0.854 & $\begin{array}{l}1.04(0.47, \\
2.31)\end{array}$ & 0.924 \\
\hline Duration, min & $16(6,32)$ & $14(6,28)$ & $\begin{array}{l}0.84(0.71 \\
1.00)\end{array}$ & 0.048 & $\begin{array}{l}0.96(0.82 \\
1.12)\end{array}$ & 0.587 \\
\hline \multicolumn{7}{|l|}{$\begin{array}{l}\text { Hypotension MAP < } \\
55 \mathrm{mmHg}\end{array}$} \\
\hline Frequency, n (\%) & $\begin{array}{l}485 \\
(65.28 \%)\end{array}$ & $\begin{array}{l}128 \\
(60.66 \%)\end{array}$ & $\begin{array}{l}0.82(0.60, \\
1.12)\end{array}$ & 0.218 & $\begin{array}{l}0.90(0.64, \\
1.26)\end{array}$ & 0.531 \\
\hline Duration, min & $2(0,5)$ & $1(0,5)$ & $\begin{array}{l}0.85(0.66, \\
1.08)\end{array}$ & 0.184 & $\begin{array}{l}0.94(0.74, \\
1.21)\end{array}$ & 0.651 \\
\hline
\end{tabular}




\begin{tabular}{|c|c|c|c|c|c|c|}
\hline \multirow[t]{2}{*}{ Outcomes } & \multirow{2}{*}{$\begin{array}{l}\text { No PACU- } \\
\text { OpResc } \\
(n=743)\end{array}$} & \multirow{2}{*}{$\begin{array}{l}\text { PACU- } \\
\text { OpResc } \\
(n=211)\end{array}$} & \multicolumn{2}{|c|}{ Unadjusted analysis } & \multicolumn{2}{|c|}{ Adjusted analysis } \\
\hline & & & $\begin{array}{l}\text { OR/IRR } \\
(95 \% \mathrm{Cl})\end{array}$ & $\begin{array}{l}\text { p- } \\
\text { value }\end{array}$ & $\begin{array}{l}\text { OR/IRR } \\
(95 \% \mathrm{Cl})\end{array}$ & $\begin{array}{l}\text { p- } \\
\text { value }\end{array}$ \\
\hline \multicolumn{7}{|c|}{$\begin{array}{l}\text { Data are expressed as frequency (prevalence in \%) or median (interquartile range (25th }-75 \text { th } \\
\text { percentile), values separated by comma). Statistical analyses were performed using negative } \\
\text { binomial regression (PACU length of stay, duration of hypotension) or multivariable logistic regression } \\
\text { (all other analyses). Odds ratios (OR) are reported for logistic regression analyses, incidence rate } \\
\text { ratios (IRR) for negative binomial regression analyses. }\end{array}$} \\
\hline \multicolumn{7}{|c|}{$\begin{array}{l}\text { MAP: Mean Arterial Pressure; PACU: Post Anesthesia Care Unit; PACU-OpResc: intravenous opioid } \\
\text { rescue analgesia in the post-anesthesia care unit; PONV: Post-Operative Nausea and vomiting. }\end{array}$} \\
\hline
\end{tabular}

In a post analysis review of the APS notes for all patients exposed to PACU-OpResc $(n=211)$, we found 168 documented APS evaluations in PACU and 128 documented PACU interventions to improve pain management. This represents a positive predictive value (PPV) of PACU-OpResc for APS evaluation and intervention of $76.3 \%$ and $60.4 \%$, respectively.

\section{Phase 2: Intervention Study}

In the post-intervention phase, 179 TEAs were placed preoperatively for surgical patients. The mean \pm SD placement rate remained consistent at $26.6 \pm 5.4$ and $25.6 \pm 6.2$ TEAs per month, across pre and post intervention periods, respectively. The average compliance with SLC documentation was $39.7 \%$ for the entire post-intervention phase. Compliance per month is presented in Fig. 2. A pre-post analysis demonstrated an absolute decrease of $8.2 \%$ in the average exposure to PACU-OpResc during the post implementation phase when compared to the preceding years (Table 3). Plotting the data using a 3sigma process control chart, we found no special causes and outliers during the post-intervention period, further supporting our findings (Fig. 3).

Table 3

PACU-OpResc rates before and after implementing sensory level check documentation

\begin{tabular}{|lccc|}
\hline \multicolumn{3}{|c|}{ PACU OpResc } & Yes \\
\hline $\begin{array}{l}\text { No } \\
\text { After SLC implementation } \\
\text { (May-November 2019) }\end{array}$ & $154(86.03 \%)$ & $25(13.97 \%)$ & 179 \\
\hline $\begin{array}{l}\text { Before SLC implementation } \\
\text { (January 2016-April 2019) }\end{array}$ & $827(77.80 \%)$ & $236(22.20 \%)$ & 1063 \\
\hline $\begin{array}{l}\text { Total } \\
\text { Data analyzed using a likelihood ratio ChiSquare test (p-value=0.009). PACU-OpResc: intravenous } \\
\text { opioid rescue analgesia in the post-anesthesia care unit; SLC: Sensory level check. }\end{array}$ & \\
\hline
\end{tabular}




\section{Discussion}

In this study, we found exposure to PACU-OpResc to be significantly associated with prolonged PACU length of stay and delayed PACU discharge. We then demonstrated the utilization of this metric in a quality improvement intervention focusing on TEA catheter placement verification.

Thoracic epidural analgesia is a widely used modality for intra/post-operative pain management in major abdominal and thoracic surgeries, recommended by enhanced recovery after surgery (ERAS) protocols for several surgical disciplines.(20-22) Thus, when offered to patients preoperatively, TEA is often presented as a superior modality to IV analgesia that may contribute to faster recovery, better overall experience and perhaps improved outcome. Our findings imply that for a substantial number of patients, inadequate TEA with PACU-OpResc requirement may hinder this process.

\section{Phase 1: Patient Centered Measure for Thoracic Epidural Analgesia Effectiveness}

The primary outcome of this study was chosen to reflect the effect of inadequate TEA on resource allocation. Taking into account that Inadequate pain management is a known factor for increased PACU length of stay(23), we theorized that exposure to PACU-OpResc, as a surrogate for inadequate TEA will prolong PACU length of stay. Indeed, our analysis, adjusted for patient and procedure specific confounders, showed that patients who required PACU-OpResc had significantly longer PACU length of stay and their discharge was more frequently delayed due to inadequate pain management. We suggest that investing resources to verify adequate TEA and catheter placement, even though it may delay the OR workflow, may prove to be cost-effective, and reduce PACU length of stay for these patients.

The definition of failed epidural analgesia varies substantially between authors and ranges from specific criteria such as failure to place a catheter at $1 \mathrm{st}$ attempt(8) to broader definitions which include "documented inability to locate the epidural space during insertion or complete lack of any surgical site sensory block following epidural bolus"(13) or "any condition during the course of treatment that requires epidural catheter replacement or the addition of another major treatment modality such as IV patientcontrolled analgesia".(7) Irrespective of nomenclature, failure to achieve adequate analgesia requiring rescue analgesic management in the immediate post-operative phase represents an undesirable outcome for the patient and could be considered a marker of TEA failure. In planning this study and intervention, we focused on establishing a framework for a learning environment that would prospectively enable feedback on the effectiveness of staged quality improvement interventions. We chose to focus on PACUOpResc as pragmatic measure that represents the overall patient experience, regardless of the reason for inadequate TEA.

The rate of PACU-OpResc of $22.1 \%$ in our center lies within the previously reported range of TEA failure rates and supports PACU-OpResc as a surrogate measure for inadequate TEA. We further demonstrated good positive predictive value of PACU-OpResc for documented evaluation by the APS team and 
documented interventions to improve TEA effectiveness in PACU. Evaluation of patients with TEA and interventions may be performed by anesthesiology teams in the PACU or the OR and may not always be documented. We, therefore, assume that our calculated PPV is an underestimation of the ability of PACUOpResc to identify cases of inadequate TEA.

\section{Phase 2: Intervention Study}

While mapping and assessing the TEA placement process for potential points of intervention, we noted that on perceived successful placement of a TEA and following the administration of a standardized test dose, a sensory level check (SLC) was not being consistently performed. Furthermore, if a SLC was performed it was not documented due to the lack of a discrete documentation element in the electronic procedure note. We focused on the SLC documentation as a first PDSA cycle, through which we could gain knowledge both on the TEA process and PACU-OpResc as its measure. Our aim was to assess firstly, compliance with SLC documentation immediately after TEA placement and secondly, the impact of SLC documentation on TEA failure rates.

Our chosen intervention formally targeted catheter location verification. However, in our center, where one team places the TEA and another uses it in the OR, we theorized that taking a patient to the OR with a proven-to-be-working TEA, may incentivize physicians to utilize it better, and relay more TEAs for intraoperative analgesia. Larsson et al(15) demonstrated that adequate sensory level after a local anesthetic bolus can be achieved in 35\% of the patients within 5-6 minutes, and in $99 \%$ of the patients within 15 minutes. As we were aware of the time constrains and pressure to quickly take patients to the OR, we deliberately did not mandate or actively promote conducting a sensory level check throughout the intervention period. Thus, we believe that a level check was conducted after placement only when OR workflow permitted it, and that our compliance rate of $39.7 \%$ with level checking and documenting adequate sensory level after TEA placement, reflects an inherent ability and resources to do so in our center. We speculate that conducting a level check as well as the ability to document it have reduced PACU-OpResc by allowing for timely manipulation of improperly placed catheters and by encouraging the intraoperative blousing and infusion through TEA by the OR team. We could neither identify alternative explanations or interventions aimed at reducing opioid administration in our PACU nor detect a significant change in our anesthesia faculty, technique or equipment during our implementation period. Trends in current anesthesia practice aimed at reducing perioperative opioid administration(24-26) may have influenced our findings. However, this would explain a gradual reduction in opioid dosing rather than a significant drop in PACU-OpResc.

\section{Limitations}

This study is a single center retrospective analysis followed by an intervention which despite being effective in our center may not be applicable elsewhere. However, we present a pragmatic, feasible metric for TEA effectiveness which may be a measure for local standard of care and management of TEAs.

There are, however, biases for using this measure. Inadequate TEA may be overestimated by including patients receiving opioids as part of their post-operative anesthetic plan due to known dependency, or for 
whom a clinical decision to not use the TEA was made. We attempted to control for this confounder by adjusting our analysis for chronic opioid use. Hypotension, a common reason to discontinue the TEA infusion was included in our secondary outcomes and did not differ between the two study groups.

Underestimation of inadequate TEA using PACU-OpResc may occur when the epidural catheter was replaced, manipulated or bolused before a patient received IV opioids, or cases in which adequate analgesia was achieved with non-opioid supplement analgesics. Nevertheless, this adds strength to PACU-OpResc as a measure of the overall process and management of TEAs, also reflecting the timely identification and correction of inadequate TEA to provide better patient care. Our results are further limited by their retrospective nature and by the possibility that factors other than our intervention are responsible for this result, despite our best efforts to eliminate such historical bias.

\section{Conclusions}

The requirement for PACU-OpResc in patients with TEA was shown to prolong PACU stay. These delays were significantly associated with inadequate pain control. This surrogate measure for TEA effectiveness can be used as a metric for ongoing quality improvement projects, reflecting not only the TEA placement but also the management and intervention to improve analgesia by the local team. A simple intervention to verify catheter location was shown to substantially reduce PACU-OpResc in this single center study. Studies to assess the generalizability of this single-center study and effects of further interventions on PACU-OpResc are necessary.

\section{List Of Abbreviations}

APS: Acute Pain Service

BMI: Body Mass Index

LOS: Length of Stay

PACU: Post Anesthesia Care Unit

PACU-OpResc: Opioid Rescue in the Post Anesthesia Care Unit

PDSA: Plan-Do-Study-Act

PPV: Positive Predictive Value

RVUs: Relative Value Units

SLC: Sensory Level Check

SPORC: Score of Prediction of postOperative Respiratory complications 
TEA: Thoracic Epidural Analgesia

\section{Declarations}

\section{Ethics approval and consent to participate:}

The study used de-identified data from the institutional Anesthesia Research Data Repository, which combines perioperative data from several hospital information management systems and was approved by the institutional review board (IRB) at Beth Israel Deaconess Medical Center (Committee on Clinical Investigations, protocol number: 2018P000666). The requirement for informed written consent was waived by the IRB.

The performance improvement intervention was determined by the institutional review board at Beth Israel Deaconess Medical Center as a quality improvement project that does not constitute human subject research (Committee on Clinical Investigations, protocol number: 2020D000036).

\section{Consent for publication:}

Not applicable

\section{Availability of data and materials:}

The datasets used and/or analysed during the current study are available from the corresponding author on reasonable request.

\section{Competing interests:}

The authors declare that they have no competing interests.

\section{Funding:}

The authors have no sources of funding to declare for this manuscript.

\section{Authors' contributions:}

$\mathrm{NL}$ : This author conceived the idea for this study, designed and carried out the analysis for the intervention study, drafted, reviewed and finalized the manuscript.

PS: This author designed and carried out the research analyses, revised and finalized the manuscript.

LZ: This author contributed to the study design and aided in drafting and revising the manuscript.

SN: This author extracted and processed the data for this study and revised the manuscript.

GK: This author contributed to the study design and aided in drafting and revising the manuscript. 
SKR: Ramachandran: This author contributed to the study design, supervised the analyses, revised and finalized the manuscript.

\section{Acknowledgements.}

The authors thank all nurses, anesthesiologists and staff members who shared the patients' care. The authors thank Amy Cohen, Director of Educational Technology, Harvard T.H. Chan School of public health for her contribution to the analysis of the QI phase.

\section{References}

1. Block BM, Liu SS, Rowlingson AJ, Cowan AR, Cowan JA, Wu CL. Efficacy of Postoperative Epidural Analgesia. J Am Med Assoc. 2003;290(18):2455-63.

2. Manion M.D. SC, Brennan Ph.D., M.D. TJ, Uzan J, Carbonnel M, Piconne O, Asmar R, et al. Thoracic Epidural Analgesia and Acute Pain Management. Anesthesiol J Am Soc Anesthesiol [Internet]. 2011 Jul 1;115(1):467-74. Available from: https://doi.org/10.1097/ALN.0b013e318220847c

3. Groeben H. Epidural anesthesia and pulmonary function. J Anesth. 2006;20(4):290-9.

4. Ali M, Winter DC, Hanly AM, O'Hagan C, Keaveny J, Broe P. Prospective, randomized, controlled trial of thoracic epidural or patient-controlled opiate analgesia on perioperative quality of life. $\mathrm{Br} \mathrm{J}$ Anaesth [Internet]. 2010;104(3):292-7. Available from: http://dx.doi.org/10.1093/bja/aeq006

5. Guay J, Kopp S. Epidural pain relief versus systemic opioid-based pain relief for abdominal aortic surgery. Cochrane database Syst Rev. 2016 Jan;(1):CD005059.

6. Guay J, Nishimori M, Kopp SL. Epidural local anesthetics versus opioid-based analgesic regimens for postoperative gastrointestinal paralysis, vomiting, and pain after abdominal surgery: A cochrane review. Anesth Analg. 2016;123(6):1591-602.

7. Ready LB. Acute pain: Lessons learned from 25,000 patients. Reg Anesth Pain Med. 1999;24(6):499505.

8. Thangamuthu a, Russell IF, Purva M. Epidural failure rate using a standardised definition. Int J Obstet Anesth [Internet]. 2013 Nov [cited 2014 Apr 3];22(4):310-5. Available from:

http://www.ncbi.nlm.nih.gov/pubmed/23932551

9. Tran DQH, Van Zundert TCRV, Aliste J, Engsusophon P, Finlayson RJ. Primary failure of thoracic epidural analgesia in training centers: The invisible elephant? Reg Anesth Pain Med. 2016;41(3):309-13.

10. Heinink TP, Baker BG, Yates VF, Addison DC, Williams JP. The effect of anaesthetist grade and frequency of insertion on epidural failure: A service evaluation in a United Kingdom teaching hospital. BMC Anesthesiol. 2015;15(1):1-7.

11. Hermanides J, Hollmann MW, Stevens MF, Lirk P. Failed epidural: Causes and management. Br J Anaesth [Internet]. 2012;109(2):144-54. Available from: http://dx.doi.org/10.1093/bja/aes214 
12. Shah AC, Nair BG, Spiekerman CF, Bollag LA. Continuous intraoperative epidural infusions affect recovery room length of stay and analgesic requirements: a single-center observational study. $\mathrm{J}$ Anesth. 2017;31(4):494-501.

13. Gleicher Y, Singer O, Choi S, McHardy P. Thoracic Epidural Catheter Placement in a Preoperative Block Area Improves Operating Room Efficiency and Decreases Epidural Failure Rate. Reg Anesth Pain Med. 2017;42(5):649-51.

14. Shah AC, Nair BG, Spiekerman CF, Bollag LA. Process Optimization and Digital Quality Improvement to Enhance Timely Initiation of Epidural Infusions and Postoperative Pain Control. Anesth Analg. 2019;128(5):953-61.

15. Larsson J, Gordh TE. Testing whether the epidural works: Too time consuming? Acta Anaesthesiol Scand. 2010;54(6):761-3.

16. Brueckmann B, Villa-Uribe JL, Bateman BT, Grosse-Sundrup M, Hess DR, Schlett CL, et al. Development and Validation of a Score for Prediction of Postoperative Respiratory Complications. Anesthesiol J Am Soc Anesthesiol [Internet]. 2013 Jun 1;118(6):1276-85. Available from: https://doi.org/10.1097/ALN.0b013e318293065c

17. Langley GJ. The Improvement Guide: A Practical Approach to Enhancing Organizational Performance [Internet]. Vol. 2nd ed. San Francisco: Jossey-Bass; 2009. Available from: http://ezpprod1.hul.harvard.edu/login?url=http://search.ebscohost.com/login.aspx? direct=true\&db=nlebk\&AN=279167\&site=ehost-live\&scope=site

18. von Elm E, Altman DG, Egger M, Pocock SJ, Gøtzsche PC VJSI. The Strengthening the Reporting of Observational Studies in Epidemiology (STROBE)statement: guidelines for reporting observational studies. J Clin Epidemiol. 2008;Apr(61(4)):344-9.

19. Ogrinc G, Davies L, Goodman D, Batalden PB, David off F SD. SQUIRE 2.0 (Standards for QUality Improvement Reporting Excellence): Revised publication guidelines from a detailed consensus process. BMJ Qual Saf. 2016;25:986-92.

20. Vukovic N, Dinic L. Enhanced recovery after surgery protocols in major urologic surgery. Front Med. 2018;5(APR):1-10.

21. Crumley S, Schraag S. The role of local anaesthetic techniques in ERAS protocols for thoracic surgery. J Thorac Dis. 2018;10(3):1998-2004.

22. Fawcett WJ, Baldini G. Optimal Analgesia During Major Open and Laparoscopic Abdominal Surgery. Anesthesiol Clin. 2015;33(1):65-78.

23. Ganter MT, Blumenthal S, Dübendorfer S, Brunnschweiler S, Hofer T, Klaghofer R, et al. The length of stay in the post-anaesthesia care unit correlates with pain intensity, nausea and vomiting on arrival. Perioper Med. 2014;3(1):1-9.

24. Clark DJ, Schumacher MA. America's Opioid Epidemic: Supply and Demand Considerations. Anesth Analg. 2017;125(5):1667-74.

25. Brandal D, Keller MS, Lee C, Grogan T, Fujimoto Y, Gricourt Y, et al. Impact of Enhanced Recovery after Surgery and Opioid-Free Anesthesia on Opioid Prescriptions at Discharge from the Hospital: A 
Historical-Prospective Study. Anesth Analg. 2017;125(5):1784-92.

26. Sultana A, Torres D, Schumann R. Special indications for Opioid Free Anaesthesia and Analgesia, patient and procedure related: Including obesity, sleep apnoea, chronic obstructive pulmonary disease, complex regional pain syndromes, opioid addiction and cancer surgery. Best Pract Res Clin Anaesthesiol [Internet]. 2017;31(4):547-60. Available from:

https://doi.org/10.1016/j.bpa.2017.11.002

\section{Figures}

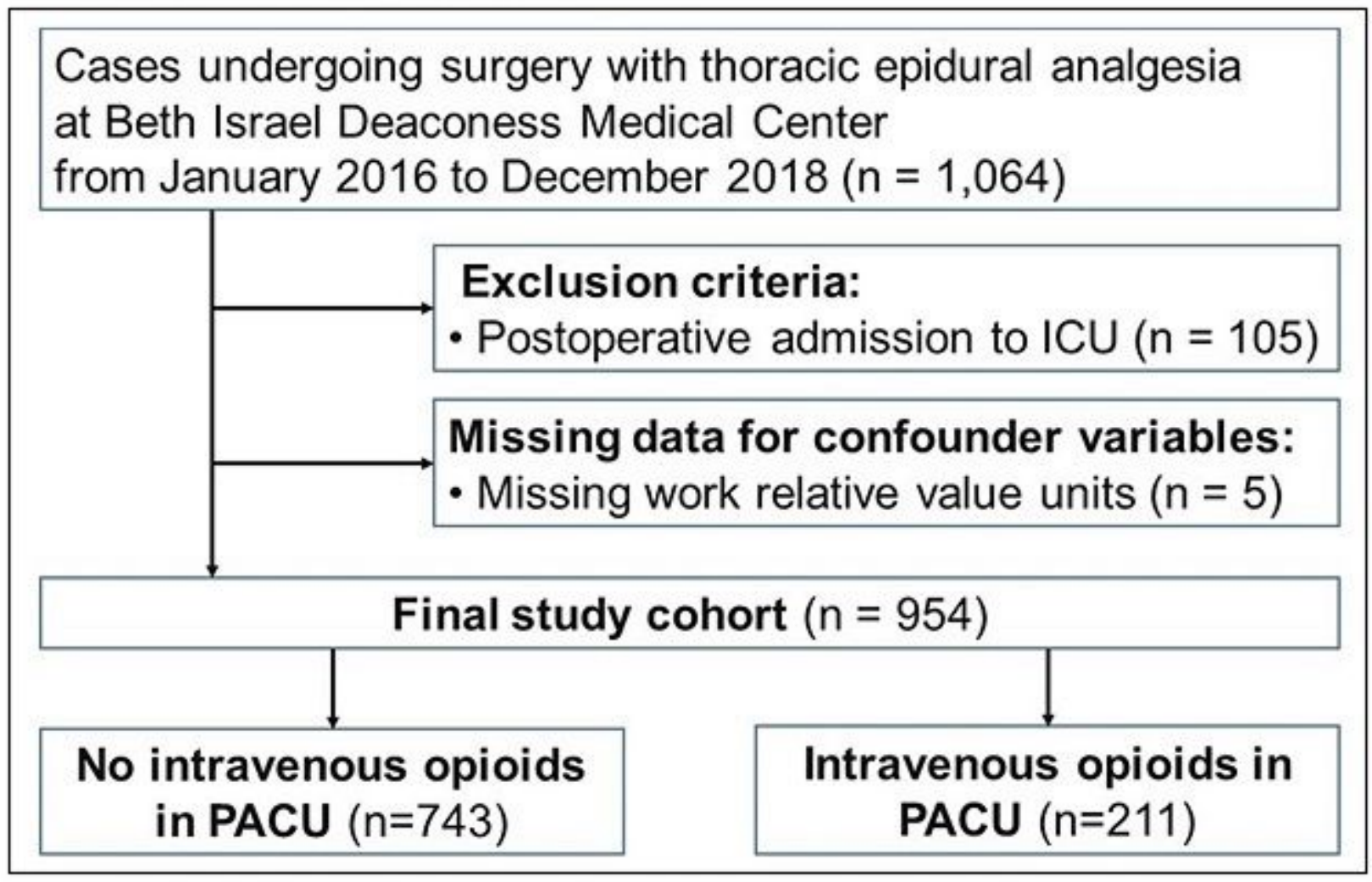

ICU: Intensive Care Unit; PACU: Post Anesthesia Care Unit.

Figure 1

Study flow diagram 


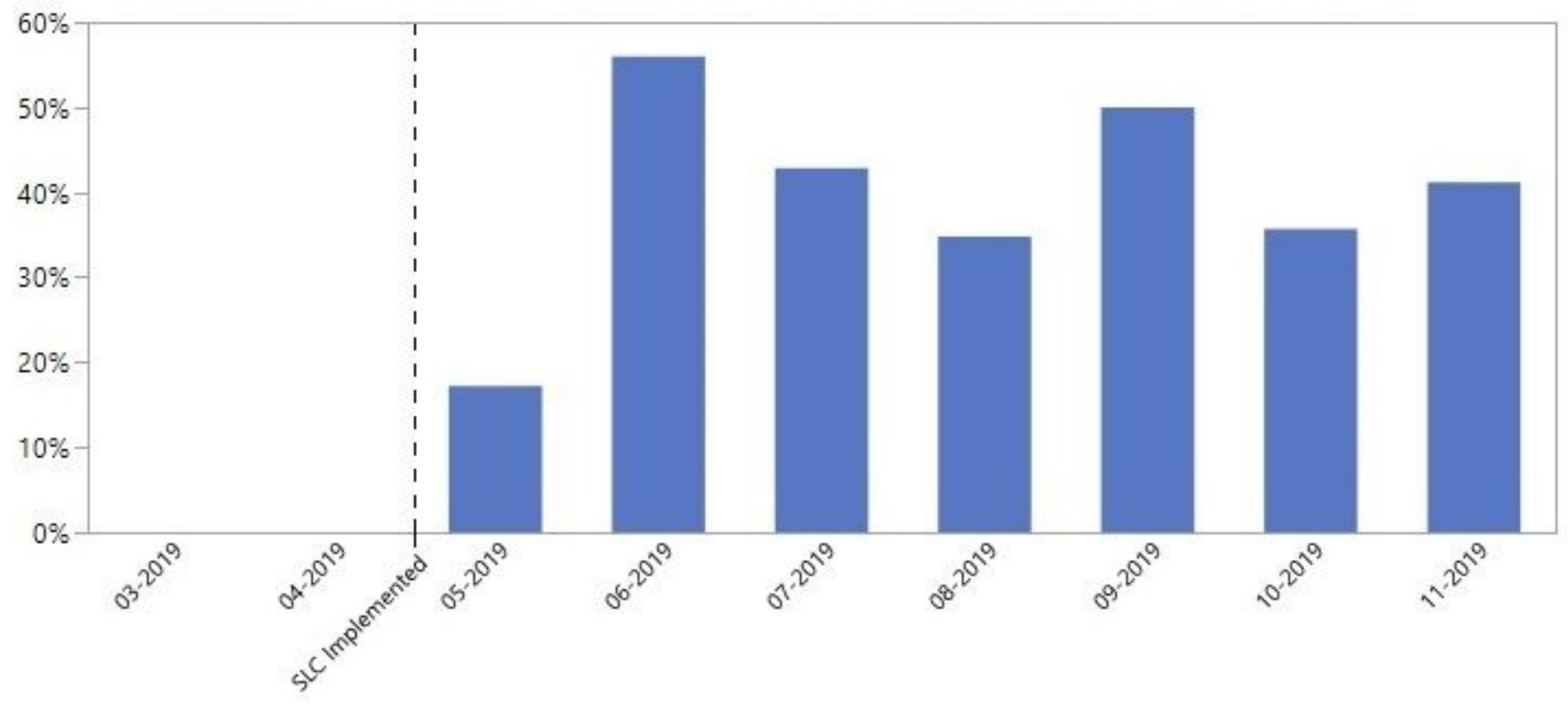

SLC: Sensory Level Check

Figure 2

Monthly rate of Thoracic Epidurals with documented Sensory Level Check. Percentage of Thoracic Epidurals with documented sensory level check following implementation of discreate SLC documentation field in the electronic procedure note. Data presented as \% [SLC] /[Total placements] per month.

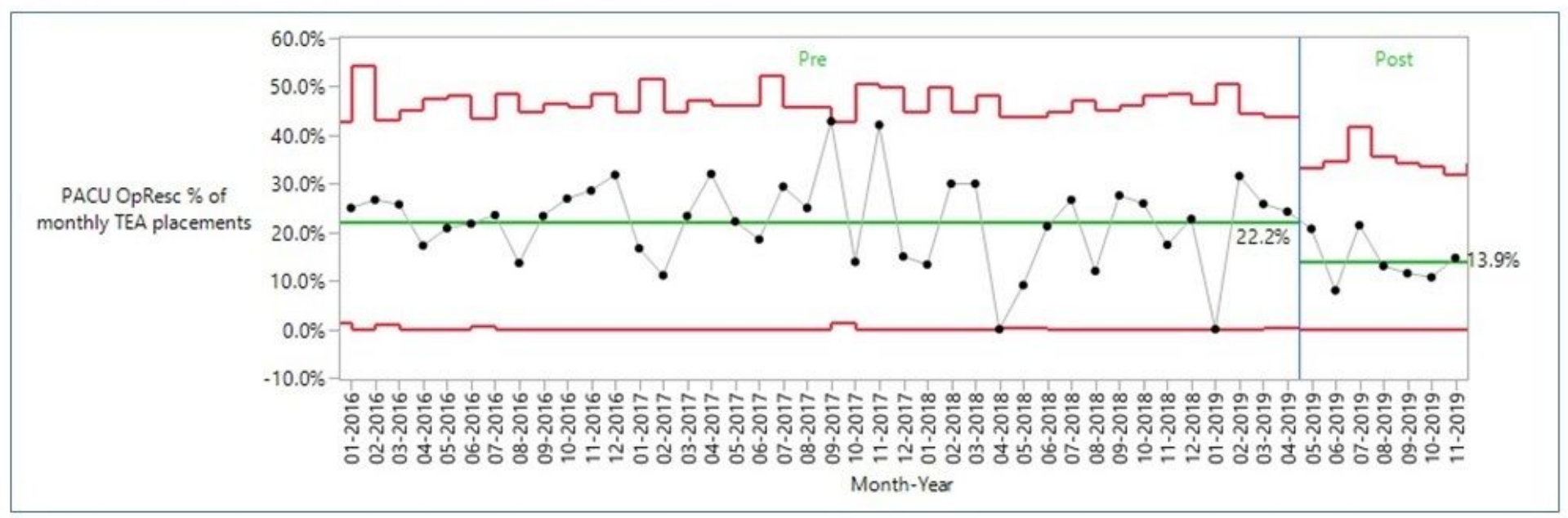

PACU: Post Anesthesia Care Unit; PACU-OpResc: intravenous opioid rescue analgesia in the post-anesthesia care unit; SLC: Sensory Level Check; TEA: Thoracic Epidural Analgesia.

\section{Figure 3}


Phased process control chart of monthly PACU-OpResc. Phased statistical process control chart (p-chart) of PACU-OpResc as a proportion of monthly TEA. Middle horizontal line reflects the weighted average PACU-OpResc rate before (22.2\%) and after (13.9\%) implementing SLC documentation. Upper and lower horizontal lines reflect the upper and lower $3 \sigma$ control limits for each month, respectively. Vertical line marks the implementation of SLC documentation (May 2019). Sample size (TEA placements) was insufficient to determine the lower control limit during April 2018 and January 2019, therefore the zeroPACU-OpResc rate in these months is not considered to be "special cause".

\section{Supplementary Files}

This is a list of supplementary files associated with this preprint. Click to download.

- Appendix01.docx 